\title{
Recruitment for Remote Decentralized Studies in Parkinson's Disease
}

\author{
Taylor L. Myers ${ }^{\mathrm{a}}$, Erika F. Augustine ${ }^{\mathrm{a}, \mathrm{b}}$, Elizabeth Baloga ${ }^{\mathrm{a}}$, Margaret Daeschler ${ }^{\mathrm{d}}$, Paul Cannon ${ }^{\mathrm{e}}$, \\ Helen Rowbotham ${ }^{\mathrm{e}}$, Eli Chanoff ${ }^{\mathrm{e}}$, 23andMe Research Team ${ }^{\mathrm{e}}$, Stella Jensen-Roberts ${ }^{\mathrm{a}}$, Julia Soto ${ }^{\mathrm{a}}$, \\ Robert G. Hollowayc ${ }^{\mathrm{c}}$, Connie Marras ${ }^{\mathrm{f}}$, Caroline M. Tanner ${ }^{\mathrm{g}, \mathrm{h}}$, \\ E. Ray Dorsey ${ }^{\mathrm{a}, \mathrm{c}}$ and Ruth B. Schneider ${ }^{\mathrm{a}, \mathrm{c}, *}$ \\ ${ }^{a}$ Center for Health + Technology, University of Rochester, Rochester, NY, USA \\ ${ }^{\mathrm{b}}$ Kennedy Krieger Institute, Baltimore, MD, USA \\ ${ }^{\mathrm{c}}$ Department of Neurology, University of Rochester, Rochester, NY, USA \\ ${ }^{d}$ Michael J. Fox Foundation for Parkinson's Research, New York, NY, USA \\ e23andMe, Inc., Sunnyvale, CA, USA \\ ${ }^{\mathrm{f}}$ The Edmond J Safra Program in Parkinson's disease, University Health Network, University of Toronto, Toronto, \\ ON, Canada \\ ${ }^{\mathrm{g}}$ Department of Neurology, Weill Institute for Neurosciences, University of California San Francisco, \\ San Francisco, CA, USA \\ ${ }^{\mathrm{h}}$ PADRECC, San Francisco VAMC, San Francisco, CA, USA
}

Accepted 11 September 2021

Pre-press 31 October 2021

\begin{abstract}
.
Background: Traditional in-person Parkinson's disease (PD) research studies are often slow to recruit and place unnecessary burden on participants. The ongoing COVID-19 pandemic has added new impetus to the development of new research models. Objective: To compare recruitment processes and outcomes of three remote decentralized observational PD studies with video visits.

Methods: We examined the number of participants recruited, speed of recruitment, geographic distribution of participants, and strategies used to enhance recruitment in FIVE, a cross-sectional study of Fox Insight participants with and without PD ( $n=203)$; VALOR-PD, a longitudinal study of $23 \mathrm{andMe}$, Inc. research participants carrying the LRRK2 G2019S variant with and without PD $(n=277)$; and AT-HOME PD, a longitudinal study of former phase III clinical trial participants with PD $(n=226)$.

Results: Across the three studies, 706 participants from 45 U.S. states and Canada enrolled at a mean per study rate of 4.9 participants per week over an average of 51 weeks. The cohorts were demographically homogenous with regard to race (over 95\% white) and level of education (over 90\% with more than a high school education). The number of participants living in primary care Health Professional Shortage Areas in each study ranged from 30.3-42.9\%. Participants reported interest in future observational (98.5-99.6\%) and interventional (76.1-87.6\%) research studies with remote video visits.

Conclusion: Recruitment of large, geographically dispersed remote cohorts from a single location is feasible. Interest in participation in future remote decentralized PD studies is high. More work is needed to identify best practices for recruitment, particularly of diverse participants.
\end{abstract}

Keywords: Parkinson's disease, telemedicine, recruitment, decentralized

\footnotetext{
*Correspondence to: Ruth Schneider, MD, University of Rochester, 265 Crittenden Blvd, Box MIND, Rochester, NY
}

14642, USA. Tel.: +1 585273 1856; E-mail: Ruth_schneider@ urmc.rochester.edu. 


\section{INTRODUCTION}

Traditional, in-person clinical research studies are plagued by slow and often unsuccessful recruitment [1]. The location of research sites, travel burden, and reliance on busy clinicians to identify potential participants [2] are all factors that contribute to recruitment difficulties. These obstacles are exacerbated in Parkinson's disease (PD) research, where physical disability, cognitive impairment, and decreased driving ability interfere with research participation $[3,4]$.

One potential solution is the decentralized research study, in which study visits occur outside of the typical clinical trial setting and data is collected directly from participants [5]. With this model, assessments may be conducted remotely and digital measures can complement traditional measures. Interest in decentralized research models has been increasing, driven not only by interest in reducing participant burden and enhancing recruitment, but also by the ongoing COVID-19 pandemic, which has delayed and interrupted clinical research [6]. One COVID19 treatment trial has already been conducted using a decentralized framework [7]. Prior remote PD research studies have demonstrated high satisfaction with and interest in this model [8]. However, remote decentralized research studies cannot rely on the same recruitment tactics as in-person studies, raising the question of how best to identify and enroll participants.

Here, we examine recruitment processes and outcomes for three remote decentralized PD research studies with the aim of jump-starting the development of best practices for future remote decentralized clinical trials.

\section{METHODS}

We examined recruitment for three independent remote decentralized, observational PD clinical research studies: Fox Insight Validation Effort (FIVE), Assessing Tele-Health Outcomes in Multiyear Extensions of Parkinson's Disease trials (ATHOME PD), and Virtual Assessment of LRRK2 carriers to Optimize Research in Parkinson's disease (VALOR-PD) (Table 1). All studies were approved by the University of Rochester Institutional Review Board. All participants provided informed consent. Detailed study methods have been previously reported for each study [9-11].

\section{Fox Insight Validation Effort (FIVE)}

FIVE was a cross-sectional study sponsored by the Michael J. Fox Foundation for Parkinson's Research (MJFF) and was designed to validate an online selfreported method for collecting PD status and other health information among participants in Fox Insight. Fox Insight has enrolled over 45,000 participants with and without PD [12]. Participants in FIVE completed two video visits conducted in real-time using video-conferencing software and study-related questionnaires in Research Electronic Data Capture (REDCap, Vanderbilt University). Included were assessments of motor function, activities of daily living, and cognition.

\section{FIVE recruitment process}

Participants with and without self-reported PD who were enrolled in Fox Insight were invited to participate (Table 1). The study aimed to recruit approximately equal numbers of participants across five strata-self-reported PD $\leq 2$ years since diagnosis, 3-5 years since diagnosis, 6-9 years since diagnosis, $\geq 10$ years since diagnosis, and without a diagnosis of PD. The Fox Insight team sent an email invitation to randomly selected participants directing them to a website to learn more about the study and provide consent to contact. A FIVE study team member then completed a pre-screening telephone call, emailed the participant an electronic consent form, and scheduled the participant for their first coordinator-led video visit. After initially not meeting our recruitment targets, we implemented several strategies to enhance recruitment: 1) increased email batch size (from 50 up to 150), 2) modified email language (emphasizing the role of participants in advancing knowledge of PD), 3) added a reminder invitation email, and 4) customized emails towards healthy controls.

\section{Virtual assessment of LRRK2 carriers to optimize research in Parkinson's disease (VALOR-PD)}

VALOR-PD is an on-going 36-month, prospective, longitudinal study of individuals with and without PD with the LRRK2 G2019S variant. The study aims to create a well-characterized, engaged, and clinical trial-ready cohort of individuals with the LRRK2 G2019S variant. VALOR-PD consists of annual video visits and study-related questionnaires in REDCap. Study procedures include assessment of motor function, exposure to environmental risk 
Table 1

Study Characteristics

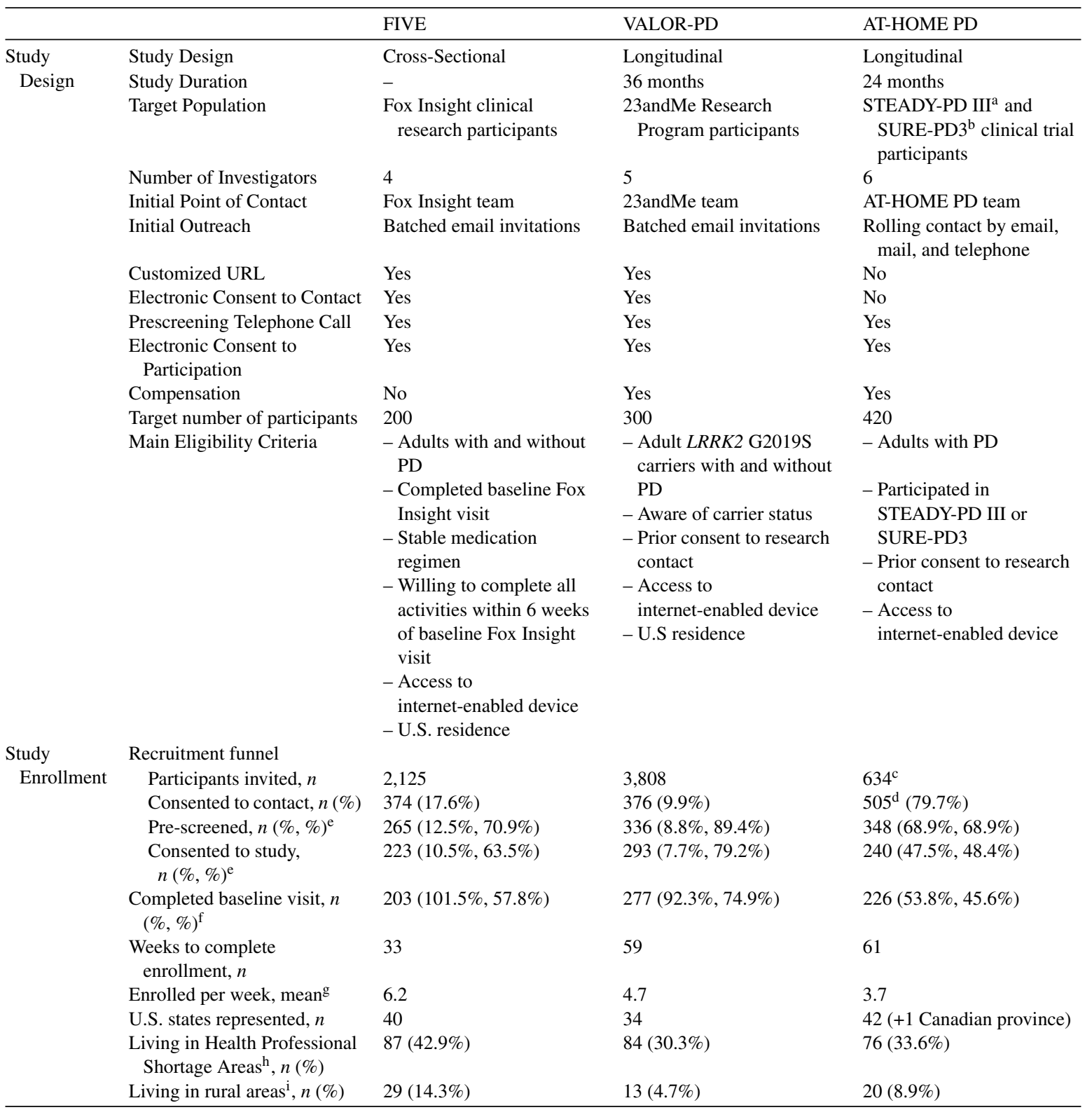

${ }^{a}$ STEADY-PDIII (Efficacy of Isradipine in Early Parkinson Disease; NCT02168842) was a phase III clinical trial of isradipine, ongoing at the time of AT-HOME PD enrollment. ${ }^{\text {b }}$ SURE-PD3 (Study of Urate Elevation in Parkinson's Disease, Phase 3; NCT02642393) was a phase III clinical trial of inosine, ongoing at the time of AT-HOME PD enrollment. ${ }^{c}$ As participation in either STEADY-PDIII or SURE-PD3 was an eligibility requirement for AT-HOME PD, the pool of all participants from the parent studies $(n=634)$ were considered to be the maximum number of individuals "invited" to participate. ${ }^{\mathrm{d}}$ All participants from the STEADY-PDIII and SURE-PD3 studies that 1$)$ were not deceased, 2) had not left the study and 3) had given consent to be contacted for future research studies $(n=505)$ were considered potentially eligible for AT-HOME PD. ${ }^{e}$ Percentages reported are (\% of participants invited, $\%$ of those consented to contact). ${ }^{\mathrm{f}}$ Percentages reported are ( $\%$ of target, $\%$ of those consented to contact). ${ }^{\mathrm{g}}$ Number of participants enrolled per week calculated by dividing the number of completed baseline visits by the number of weeks to complete enrollment. ${ }^{\text {h}}$ Participants were determined to live in a Health Professional Shortage Area (HPSA) if their mailing address zip code was included on the list of all U.S. HPSA zip codes, as determined by the Centers for Medicare \& Medicaid Services. ${ }^{i}$ Participants were determined to live in a rural area if their mailing address zip code was included on the list of all U.S. Federal Office of Rural Health Policy-eligible zip codes, as determined by the Health Resources \& Services Administration. 
factors, activities of daily living, cognition, sleep, smell, and mood.

\section{VALOR-PD recruitment process}

LRRK2 G2019S carriers with and without PD who received genetic testing through $23 \mathrm{andMe}$, a personal genetics company, and opted-in to contact regarding research opportunities, were invited to participate (Table 1$)$. Enrollment was stratified by age $(<60$ years old and $\geq 60$ years old) and by self-reported PD status, with the goal of recruiting approximately 25 participants with $\mathrm{PD}<60$ years old, 25 participants with $\mathrm{PD} \geq 60$ years old, 125 participants without PD $<60$ years old, and 125 participants without PD $\geq 60$ years old. Initial recruitment procedures were identical to FIVE except up to three emails were sent - an initial invitation and two reminder emails. To speed recruitment, we made adaptations, including 1) increasing the email batch size (from 50 to 120) and 2) modifying the email language to target individuals without PD.

\section{Assessing tele-health outcomes in multiyear extensions of PD trials (AT-HOME PD)}

AT-HOME PD is an on-going 24-month, longitudinal follow-up study of two PD clinical trial cohorts-STEADY-PD III (a study of isradipine) [13] and SURE-PD3 (a study of inosine) [14]. The study aims to establish the infrastructure for the remote follow-up of clinical trial participants, enable objective assessment of disease in the home, and develop new PD digital biomarkers. AT-HOME PD has three principal components: 1) annual video visits, 2) quarterly smartphone-based assessments of tremor, tapping, and gait/balance through the mPower 2.0 Parkinson's smartphone application, and 3) quarterly web-based surveys through Fox Insight.

\section{AT-HOME PD recruitment process}

STEADY-PD III and SURE-PD3 participants who consented to future research contact were invited to participate (Table 1). The AT-HOME PD team attempted to contact potential participants by all available means of communication (phone, mail, email) on a rolling basis. After completing a prescreening telephone call, participants were emailed an electronic consent form and scheduled for a coordi nator-led video visit. Initial adaptations to enhance recruitment included calling outside of standard business hours and the addition of recruitment emails and letters. We also used several strategies to try to engage potential participants through STEADYPD III and SURE-PD3 sites. These included asking STEADY-PD III and SURE-PD3 investigators and coordinators to contact study participants directly, publishing an AT-HOME PD study summary to the Parkinson's Study Group website, sending informational AT-HOME PD cards to sites for distribution, and holding a teleconference featuring the principal investigators of STEADY-PD III and SURE-PD3 to discuss AT-HOME PD with attendees.

\section{Analysis}

We used descriptive statistics to describe multiple different recruitment parameters in each study, including number enrolled, time to complete enrollment, rate of enrollment, and geographic distribution of participants.

\section{RESULTS}

\section{Success of recruitment}

All three studies have completed enrollment; FIVE was the only study to meet its pre-specified enrollment target. FIVE enrolled 203 (159 with and 44 without self-reported PD), VALOR-PD enrolled 277 (60 with and 217 without self-reported PD), and AT-HOME PD enrolled 226 participants (124 from STEADY-PDIII and 102 from SURE-PD3; Table 1). In FIVE, of those who consented to contact, $70.9 \%$ completed the pre-screen, $63.5 \%$ (excluding 23 ineligible individuals) consented to study participation, and $57.8 \%$ (excluding 23 ineligible individuals) completed the baseline visit. In VALOR-PD, of those who consented to contact, $89.4 \%$ completed the prescreen, $79.2 \%$ (excluding 6 ineligible individuals) consented to study participation, and $74.9 \%$ (excluding 6 ineligible individuals) completed the baseline visit. In AT-HOME PD, of those who consented to contact during parent study participation, $68.9 \%$ completed the pre-screen, $48.4 \%$ (excluding 9 ineligible individuals) consented to study participation, and $45.6 \%$ (excluding 9 ineligible individuals) completed the baseline visit.

\section{Reasons for non-participation}

Reasons for non-participation, which were not systematically assessed, varied among the studies (Fig. 1). Across the three studies, 38 (range 6-23) were deemed ineligible. Among the eligible 

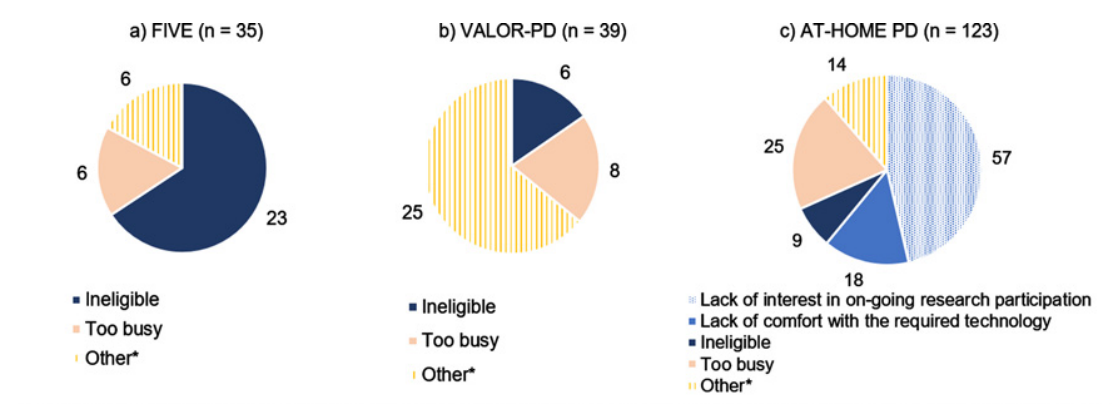

"Other reasons for non-participation included privacy concerns, lack of comfort with the required technology, and insufficient compensation (VALOR-PD)

Fig. 1. Reasons for non-participation, by study.

participants for whom reasons for non-participation were known, the most common reasons were being too busy $(6 / 12,50.0 \%)$ in FIVE, being too busy $(8 / 33,24.2 \%)$ in VALOR-PD, and lacking interest in continued research participation $(57 / 114,50.0 \%)$ in AT-HOME PD.

\section{Speed of recruitment and impact of adaptations}

Study enrollment windows were a mean (range) 51 (33-61) weeks and the mean (range) number enrolled per week was 4.9 (3.7-6.2) (Table 1, Fig. 2). In FIVE, after changing the invitation email language and adding a reminder email, the weekly average consent to contact rate increased from $8 \%$ over the first six weeks to $19 \%$ over the subsequent 25 weeks. In AT-HOME PD, after two months of phone recruitment, only $29.9 \%$ of potential participants had been pre-screened, prompting the addition of emails and mailers. After adding emails and mailers, the mean number of pre-screens per week increased from 4.7 in the first 12 weeks to 14 in the subsequent three weeks. In VALOR-PD, the average email click rate of opened emails was $24 \%$ over the first five months and after adding language targeting individuals without $\mathrm{PD}$, it did not improve.

\section{Geographic distribution of participants}

The geographic distribution of participants for each study was widespread with 45 states (range 3442 states) and one Canadian province represented across the studies (Fig. 3). No participants were recruited from five states-Alaska, Idaho, North Dakota, Kansas, and Delaware-in any of the three studies. The number of participants living in Health Professional Shortage Areas, which in this case refers to U.S. zip codes with shortages of primary care providers, ranged from $30.3-42.9 \%$ (Table 1).

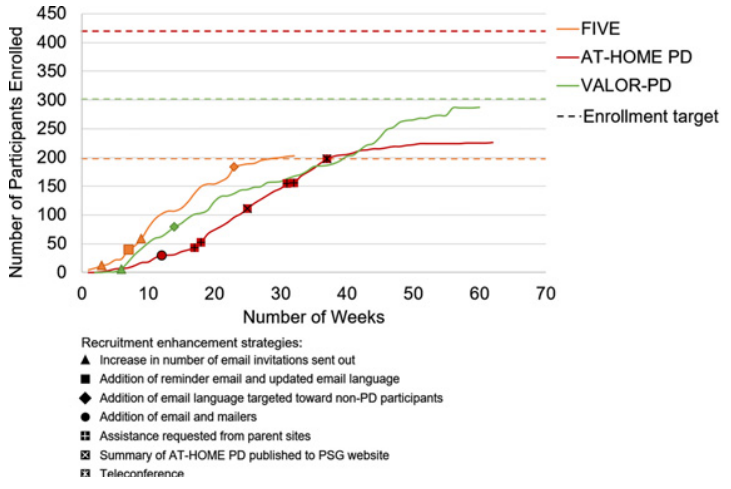

Fig. 2. Enrollment timeline, by study.

\section{Cohort demographics}

By design, the cohorts differed substantially in the percentage of individuals with PD-AT-HOME PD has all PD participants (100\%), FIVE had a large proportion of PD participants (78.3\%), and VALOR-PD is predominantly comprised of individuals genetically at-risk for PD with a small proportion of PD participants (21.7\%). Additional cohort demographics are provided in Table 2.

\section{Satisfaction and future research participation}

Participants in all three studies were highly satisfied with video visits ( $>97 \%$ overall satisfaction in all studies) (Fig. 4). Across the studies, the most frequent concerns about video visits related to confidentiality, which occurred in a minority of participants -FIVE (6.6\%), VALOR-PD (3.5\%), and AT-HOME PD (3.0\%). Questions asked regarding interest in future research opportunities were similar but not the same across the studies. Most participants in FIVE (98.5\%) and VALOR-PD (99.6\%) reported being willing to participate in future observational research 


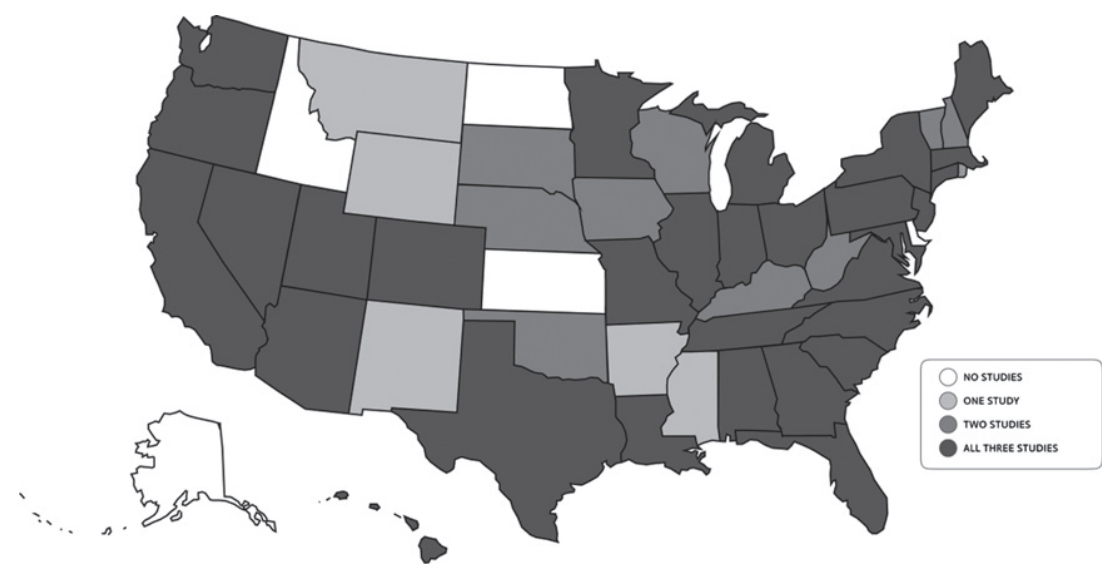

Fig. 3. Geographic distribution of participants.

Table 2

Cohort Demographics and Clinical Characteristics

\begin{tabular}{|c|c|c|c|c|c|}
\hline & \multicolumn{2}{|c|}{ FIVE } & \multicolumn{2}{|c|}{ VALOR-PD } & \multirow{2}{*}{$\begin{array}{c}\text { AT-HOME PD } \\
\text { PD } \\
(n=226)\end{array}$} \\
\hline & $\begin{array}{c}\text { PD } \\
(n=159)\end{array}$ & $\begin{array}{c}\text { Controls } \\
(n=44)\end{array}$ & $\begin{array}{l}\text { LRRK2 with PD } \\
\quad(n=60)\end{array}$ & $\begin{array}{c}\text { LRRK2 without PD } \\
\quad(n=217)\end{array}$ & \\
\hline Age, mean (SD) & $66.7(8.9)$ & $62.2(11.7)$ & $67.8(8.4)$ & $53.7(15.1)$ & $65.9(9.4)$ \\
\hline Non-white, $n(\%)$ & $6(3.9 \%)$ & $<5^{\mathrm{a}}$ & $<5^{\mathrm{a}}$ & $<5^{\mathrm{a}}$ & $10(4.4 \%)$ \\
\hline Latino/Hispanic, $n(\%)$ & $<5$ & $<5$ & $<5$ & $16(7.4 \%)$ & $6(2.7 \%)$ \\
\hline Male, $n(\%)$ & $97(61.0 \%)$ & $22(50.0 \%)$ & $29(48.3 \%)$ & $89(41.4 \%)$ & $136(60.2 \%)$ \\
\hline Education $>$ High School, $n(\%)$ & $143(89.9 \%)$ & $42(94.5 \%)$ & $59(98.3 \%)$ & $215(99.0 \%)$ & $213(94.2 \%)$ \\
\hline Montreal Cognitive Assessment, mean (SD) & $25.3(3.6)$ & $26.5(2.7)$ & $27.1(2.2)$ & $27.6(2.1)$ & $27.9(2.0)$ \\
\hline Modified Hoehn \& Yahr stage, median (min-max) & $2(0-5)$ & $0(0-4)$ & $2(1-5)$ & $0(0-2)$ & $2(0-3)$ \\
\hline Taking levodopa, $n(\%)$ & $124(78.0 \%)$ & $3(6.8 \%)$ & $53(88.3 \%)$ & $0(0.0 \%)$ & $195(86.2 \%)$ \\
\hline
\end{tabular}

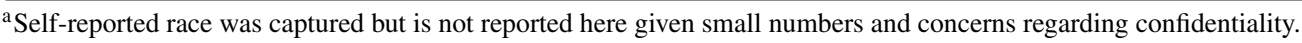

studies that include video visits. Most participants in FIVE (76.1\%) and VALOR-PD (87.6\%) reported they would be willing to participate in future clinical trials that include video visits. Compared with studies with all in-person visits, the majority of those willing to participate in future research were more interested in participating in research studies with video visits, both observational (FIVE $(62 \%)$ and VALOR-PD (65\%)) and interventional (FIVE (52\%) and VALOR-PD (60\%)). These questions were not asked of AT-HOME PD participants. Among ATHOME PD participants, compared to a trial without any remote assessments, $65 \%$ were more willing to participate in a future clinical trial with some remote assessments and $40 \%$ with all remote assessments.

\section{DISCUSSION}

These three studies-FIVE, VALOR-PD, and ATHOME PD - demonstrate that nationwide recruitment of participants with PD, without PD, and with

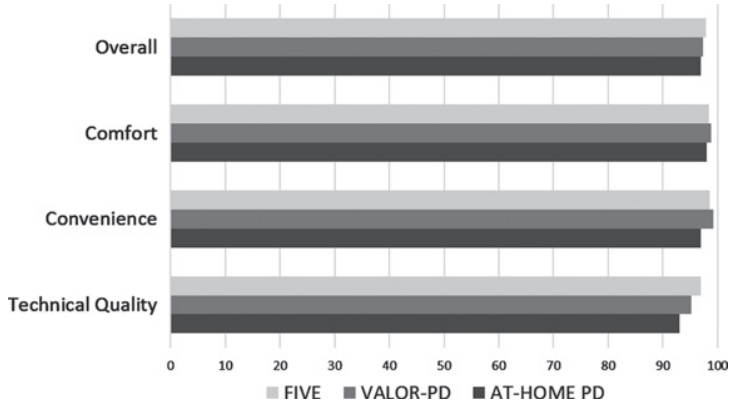

Fig. 4. Percentage of participants "satisfied" or "highly satisfied" with various aspects of virtual visits.

a genetic risk factor for PD into remote decentralized research studies is feasible and well-liked by participants. These results inform and support the future of remote decentralized research studies in PD and likely beyond, however, more work is needed to identify best practices for recruitment.

Remote studies enable research to be conducted using a single IRB and from a single location, 
which in turn reduces costs, complexity, and enrollment time, while also affording greater flexibility. In the three studies presented, a mean (range) of 4.9 (3.7-6.2) participants were enrolled per week. In-person PD research studies may exceed these enrollment rates, however, they require a large number of sites and substantial resources. For example, OBSERVE-PD (an in-person, cross-sectional study of individuals with $\mathrm{PD}$ ) recruited an average of 0.4 participants per week at each of its 128 sites during 48.5 weeks of recruitment $[15,16]$ and PPMI (an in-person, longitudinal, observational study of individuals with and without PD including blood, CSF and tissue sampling as well as neuroimaging) enrolled an average of 1 PD participant and 0.5 nonPD participants per month at each of its 24 sites [17]. While it is not realistic to compare recruitment between observational and interventional studies, as a reference, STEADY-PD III, with its comprehensive recruitment "tool kit" for sites, averaged 7.0 participants enrolled per week for twelve months across its 57 sites combined [18]. We also recognize that there are trade-offs with remote video visits that should be considered; some testing cannot be performed, and biospecimen collection is limited to what participants can collect themselves.

All three studies had expansive geographical spread despite being conducted from Rochester, NY. The VALOR-PD cohort represented the fewest states (34) and had the lowest percentage of participants residing in primary care Health Professional Shortage Areas (HPSA) (30\%). This is likely because only individuals interested in genetic testing and, with few exceptions, possessing disposable/higher income entered into 23 andMe's pool of potential participants. In contrast, FIVE had participants from the most states (40) and the highest percentage of participants living in primary care HPSAs (42.9\%). The potential geographic spread for AT-HOME PD participants was dictated by the distribution of parent study sites (which were located in 36 states, Puerto Rico, and one Canadian province). In order to achieve comparable geographic spread, in-person studies require multiple geographically dispersed sites. For example, PPMI has 34 sites (mean 20.1 participants per site) [17], STEADY-PDIII 56 sites (mean 6.0 participants per site) [13], SURE-PD3 54 sites (mean 5.5 participants per site) [14], and the LRRK2 Cohort Consortium 20 sites (mean 75.3 participants per site) [19]. Furthermore, the nature of in-person studies restricts participation to individuals living in proximity to a research center. While anecdotal, we note that we conducted visits with participants who were located in offices, parks, parking lots, and even on boats. By shifting research from clinics to participant-selected locations, decentralized studies allow for enrollment of individuals without prior access to research centers.

Across the three studies, five states go unrepresented: Alaska, Idaho, North Dakota, Kansas, and Delaware. All of these states but Delaware are among the ten least populated states in the U.S. Difficulties recruiting from these states may reflect the persistence of a geographically-defined digital divide. The digital divide reflects differential access to technologies based on socio-economic, geographic, or other factors [20]. Indeed, only $63 \%$ of rural households and $56 \%$ of individuals making $\$ 30,000$ or less per year have access to broadband [21]. Across the three studies only $4.7 \%-14.3 \%$ of participants were located in rural zip codes (defined as having $>50 \%$ of its population residing in either a Non-Metro County and/or a rural Census Tract [22]) and 30.3-42.9\% of participants were located in primary care HPSA. We used the latter as a proxy to identify underserved areas but recognize that a broad range of social determinants of health influence access to healthcare beyond the availability of providers [23]. The COVID-19 pandemic has forced the transition of many aspects of daily life, education [24], clinical care [25], and research online, highlighting the critical need for expansion of broadband internet access to enable equal opportunity for participation in future studies.

While remote studies stand to reduce geographic barriers to research participation, the three studies examined here failed to recruit a racially, ethnically, or educationally diverse sample of participants. Across the three studies, less than 5\% were nonwhite, less than $7.5 \%$ were Hispanic/Latino, and 90.7-98.5\% had more than a high school education. In part, this reflects lack of diversity of the population pools from which each study recruited. The challenge of recruiting diverse participants is not unique to remote studies [26]. Between 1985 and 2007 , only $17 \%$ of PD trials reported the race and ethnicity of participants [27]. Of those that did report this information, only $0.9 \%$ of participants were Black, 0.7\% Asian, and 1.1\% Hispanic [27]. In addition to the expansion of broadband internet access, possible solutions include collaboration with community groups and trusted organizations and geotargeted Facebook and Google recruitment efforts. The latter was used by Fox Insight to boost recruitment of non-white and Hispanic individuals [28]. 
Once individuals are identified, the free provision of devices and data plans may enable participation of those otherwise unable to participate. More research is needed in this area in particular to develop best practices.

While each study recruited a large number of participants, two of the three studies failed to meet pre-specified recruitment targets. FIVE was the only study to meet (and exceed) its target enrollment and featured the fastest recruitment. We posit that this was because individuals were already familiar with online PD research, initial contact came from a familiar source (MJFF), the duration of commitment was short, and there was an easily accessible website with study information. Recruitment does not appear to have been hindered by the lack of compensation. Of those who consented to study team contact, VALORPD was the most successful in reaching participants for a pre-screen-a critical step in the recruitment process-and converting consents to contact into enrollment. This may reflect enthusiasm for PD prevention research studies in a population with a genetic risk factor for PD. In contrast, AT-HOME PD, may have been hindered by a lack of email and mailing addresses or updated phone numbers, individuals' unfamiliarity with the study team, disappointment with the negative results of the parent clinical trials, and/or lack of enthusiasm for the study's aims. We saw an increase in response rate after collaborating with local parent study sites, suggesting that better cooperation from the outset may have enhanced enrollment. In addition, while FIVE and VALORPD drew from pools of individuals who were already engaged in online research, AT-HOME PD drew from a pool of individuals engaged in a traditional inperson research study.

A comparison of the three studies presented here offers preliminary suggestions for how to successfully recruit individuals into remote PD studies. One, initial contact about the study should come from a familiar source where possible. Large-scale outreach, such as podcasts and webinars publicized by a well-known organization in the PD community (e.g. MJFF), may be effective, as evidenced by the efficient recruitment in STEADY-PDIII [18]. Second, recruitment materials should be multimodal. Recommended modalities to include are online (website), phone, email, mail, and webinar/podcast to reach the broadest possible audience. Third, as with traditional studies, recruitment success should be tracked and continuously evaluated with strategies adapted as necessary. For example, in AT-HOME PD, we identified a low rate of successful telephone contacts and successfully adapted our approach to include email and mail-based recruitment strategies.

Our study is not without limitations. First, all three study designs featured a pre-specified pool of potential participants who were already engaged in some form of research. As such, our results may not be generalizable to the identification of potential participants from the general population. Second, none of the studies systematically tracked reasons for nonparticipation and analyses on the impact of changes in recruitment strategies were not pre-planned. Third, our analyses are based on only three studies. Given the large number of variables that may impact recruitment, our outcomes may reflect other aspects of study design unrelated to the remote decentralized design. Lastly, we do not have recruitment outcome data to enable direct comparisons with traditional, in-person research studies. Nonetheless, our experiences may inform recruitment strategies for future PD clinical trials.

Recruitment of geographically widespread individuals with and without PD into remote decentralized observational PD studies is feasible. Notably, initial satisfaction with the examined studies was uniformly high and participants expressed interest in participating in future remote PD studies supporting future expansion of such models. However, two of the studies failed to meet recruitment targets and none of the studies were racially, ethnically, or educationally diverse. More work is needed to develop best recruitment strategies, particularly in the recruitment of Black and Hispanic/Latino participants.

\section{ACKNOWLEDGMENTS}

Research reported in this publication was supported by the National Institute of Neurological Disorders and Stroke of the National Institutes of Health under award numbers P50NS108676 and U01NS107009 as well as the Michael J. Fox Foundation for Parkinson's Research under award number 15983.

We would like to thank the research participants and employees of 23andMe for making this work possible. The following members of the 23andMe Research Team contributed to this study: Michelle Agee, Stella Aslibekyan, Adam Auton, Elizabeth Babalola, Robert K. Bell, Jessica Bielenberg, Katarzyna Bryc, Emily Bullis, Briana Cameron, Daniella Coker, Gabriel Cuellar Partida, Devika 
Dhamija, Sayantan Das, Sarah L. Elson, Teresa Filshtein, Kipper Fletez-Brant, Pierre Fontanillas, Will Freyman, Pooja M. Gandhi, Karl Heilbron, Barry Hicks, David A. Hinds, Karen E. Huber, Ethan M. Jewett, Yunxuan Jiang, Aaron Kleinman, Katelyn Kukar, Vanessa Lane, Keng-Han Lin, Maya Lowe, Marie K. Luff, Jennifer C. McCreight, Matthew H. McIntyre, Kimberly F. McManus, Steven J. Micheletti, Meghan E. Moreno, Joanna L. Mountain, Sahar V. Mozaffari, Priyanka Nandakumar, Elizabeth S. Noblin, Jared O'Connell, Aaron A. Petrakovitz, G. David Poznik, Morgan Schumacher, Anjali J. Shastri, Janie F. Shelton, Jingchunzi Shi, Suyash Shringarpure, Chao Tian, Vinh Tran, Joyce Y. Tung, Xin Wang, Wei Wang, Catherine H. Weldon, Peter Wilton.

\section{CONFLICT OF INTEREST}

Paul Cannon, Helen Rowbotham, Eli Chanoff and the 23andMe Research Team are employed by and hold stock or stock options in 23andMe, Inc. Margaret Daeschler was formerly employed by the Michael J. Fox Foundation for Parkinson's Research.

\section{REFERENCES}

[1] McDonald AM, Knight RC, Campbell MK, Entwistle VA, Grant AM, Cook JA, Elbourne DR, Francis D, Garcia J, Roberts I, Snowdon C (2006) What influences recruitment to randomised controlled trials? A review of trials funded by two UK funding agencies. Trials 7, 9 .

[2] Newington L, Metcalfe A (2014) Factors influencing recruitment to research: Qualitative study of the experiences and perceptions of research teams. BMC Med Res Methodol 14, 10.

[3] Crizzle AM, Classen S, Uc EY (2012) Parkinson disease and driving: An evidence-based review. Neurology 79, $2067-$ 2074.

[4] Bouca-Machado R, Maetzler W, Ferreira JJ (2018) What is functional mobility applied to Parkinson's disease? $J$ Parkinsons Dis 8, 121-130.

[5] Dorsey ER, Kluger B, Lipset CH (2020) The new normal in clinical trials: Decentralized studies. Ann Neurol 88, 863-866.

[6] Papa SM, Brundin P, Fung VSC, Kang UJ, Burn DJ, Colosimo C, Chiang HL, Alcalay RN, Trenkwalder C; MDS-Scientific Issues Committee (2020) Impact of the COVID-19 pandemic on Parkinson's disease and movement disorders. Mov Disord 35, 711-715.

[7] Boulware DR, Pullen MF, Bangdiwala AS, Pastick KA, Lofgren SM, Okafor EC, Skipper CP, Nascene AA, Nicol MR, Abassi M, Engen NW, Cheng MP, LaBar D, Lother SA, MacKenzie LJ, Drobot G, Marten N, Zarychanski R, Kelly LE, Schwartz IS, McDonald EG, Rajasingham R, Lee TC, Hullsiek KH (2020) A randomized trial of hydroxychloroquine as postexposure prophylaxis for Covid-19. N Engl $J$ Med 383, 517-525.
[8] Shprecher D, Noyes K, Biglan K, Wang D, Dorsey ER, Kurlan R, Adams MJ (2012) Willingness of Parkinson's disease patients to participate in research using internetbased technology. Telemed J E Health 18, 684-687.

[9] Schneider RB, Omberg L, Macklin EA, Daeschler M, Bataille L, Anthwal S, Myers TL, Baloga E, Duquette S, Snyder P, Amodeo K, Tarolli CG, Adams JL, Callahan KF, Gottesman J, Kopil CM, Lungu C, Ascherio A, Beck JC, Biglan K, Espay AJ, Tanner C, Oakes D, Shoulson I, Novak D, Kayson E, Ray Dorsey E, Mangravite L, Schwarzschild MA, Simuni T, Parkinson Study Group AT-HOME PD Investigators (2021) Design of a virtual longitudinal observational study in Parkinson's disease (AT-HOME PD). Ann Clin Transl Neurol 8, 308-320.

[10] Schneider RB, Myers TL, Rowbotham HM, Luff MK, Amodeo K, Sharma S, Wilson R, Jensen-Roberts S, Auinger P, McDermott MP, Alcalay RN, Biglan K, Kinel D, Tanner C, Winter-Evans R, Augustine EF, Cannon P, andMe Research T, Holloway RG, Dorsey ER (2020) A virtual cohort study of individuals at genetic risk for Parkinson's disease: Study protocol and design. J Parkinsons Dis 10, 1195-1207.

[11] Myers TL, Tarolli CG, Adams JL, Barbano R, Cristina Gil-Díaz M, Spear KL, Lowell J, Daeschler M, Riley L, Amondikar N, Auinger P, Marras C, Tanner CM, Ray Dorsey E, Schneider RB (2021) Video-based Parkinson's disease assessments in a nationwide cohort of Fox Insight participants. Clin Parkinsonism Relat Disord 4, 100094.

[12] Smolensky L, Amondikar N, Crawford K, Neu S, Kopil CM, Daeschler M, Riley L, 23andMe Research Team, Brown E, Toga AW, Tanner C (2020) Fox Insight collects online, longitudinal patient-reported outcomes and genetic data on Parkinson's disease. Sci Data 7, 67.

[13] Parkinson Study Group STEADY-PD III Investigators (2020) Isradipine versus placebo in early Parkinson disease: A randomized trial. Ann Intern Med 172, 591-598.

[14] ClinicalTrials.gov (2000) Identifier NCT02642393, Study of Urate Elevation in Parkinson's Disease, Phase 3 (SUREPD3). Available from: https://clinicaltrials.gov/ct2/show/ NCT02642393

[15] Fasano A, Fung VSC, Lopiano L, Elibol B, Smolentseva IG, Seppi K, Takats A, Onuk K, Parra JC, Bergmann L, Sail K, Jalundhwala Y, Pirtosek Z (2019) Characterizing advanced Parkinson's disease: OBSERVE-PD observational study results of 2615 patients. BMC Neurol 19, 50.

[16] Australian and New Zealand Clinical Trials Registry, NHMRC Clinical Trials Centre, University of Sydney, Sydney, Australia.

[17] Marek K, Chowdhury S, Siderowf A, Lasch S, Coffey CS, Caspell-Garcia C, Simuni T, Jennings D, Tanner CM, Trojanowski JQ, Shaw LM, Seibyl J, Schuff N, Singleton A, Kieburtz K, Toga AW, Mollenhauer B, Galasko D, Chahine LM, Weintraub D, Foroud T, Tosun-Turgut D, Poston K, Arnedo V, Frasier M, Sherer T, Parkinson's Progression Markers Initiative (2018) The Parkinson's Progression Markers Initiative (PPMI) - establishing a PD biomarker cohort. Ann Clin Transl Neurol 5, 1460-1477.

[18] Berk S, Greco BL, Biglan K, Kopil CM, Holloway RG, Meunier C, Simuni T (2017) Increasing efficiency of recruitment in early Parkinson's disease trials: A case study examination of the STEADY-PD III Trial. J Parkinsons Dis 7, 685-693.

[19] Marras C, Alcalay RN, Caspell-Garcia C, Coffey C, Chan P, Duda JE, Facheris MF, Fernandez-Santiago R, RuizMartinez J, Mestre T, Saunders-Pullman R, Pont-Sunyer 
C, Tolosa E, Waro B, Consortium LC (2016) Motor and nonmotor heterogeneity of LRRK2-related and idiopathic Parkinson's disease. Mov Disord 31, 1192-1202.

[20] Norris P (2001) Digital Divide: Civic Engagement, Information Poverty, and the Internet Worldwide, Cambridge University Press, Cambridge.

[21] Center PR, Internet/Broadband Fact Sheet, https://www. pewresearch.org/internet/fact-sheet/internet-broadband/\# who-has-home-broadband,

[22] Federal Office of Rural Health Policy (FORHP). Health Resources \& Services Administration. (2021) FORHP Eligible ZIP Codes. Available from: https://www.hrsa.gov/ rural-health/about-us/definition/datafiles.html

[23] Streeter RA, Snyder JE, Kepley H, Stahl AL, Li T, Washko MM (2020) The geographic alignment of primary care Health Professional Shortage Areas with markers for social determinants of health. PLoS One 15, e231443.

[24] Li C, Lalani F (2020) The COVID-19 pandemic has changed education forever. This is how, World Economic Forum, https://www.weforum.org/agenda/2020/04/coronaviruseducation-global-covid19-online-digital-learning, Posted April 29, 2020, Accessed February 3, 2021.
[25] Larson DN, Schneider RB, Simuni T (2021) A new era: The growth of video-based visits for remote management of persons with Parkinson's disease. J Parkinsons Dis 11(s1), S27-S34

[26] Tilley BC, Mainous AG, 3rd, Elm JJ, Pickelsimer E, Soderstrom LH, Ford ME, Diaz VA, Siminoff LA, Burau K, Smith DW (2012) A randomized recruitment intervention trial in Parkinson's disease to increase participant diversity: Early stopping for lack of efficacy. Clin Trials 9, 188-197.

[27] Schneider MG, Swearingen CJ, Shulman LM, Ye J, Baumgarten M, Tilley BC (2009) Minority enrollment in Parkinson's disease clinical trials. Parkinsonism Relat Disord 15, 258-262.

[28] Dobkin RD, Amondikar N, Kopil C, Caspell-Garcia C, Brown E, Chahine LM, Marras C, Dahodwala N, Mantri S, Standaert DG, Dean M, Shoulson I, Marek K, Katz A, Korell M, Riley L, Tanner CM, Fox Insight S (2020) Innovative recruitment strategies to increase diversity of participation in Parkinson's disease research: The Fox Insight Cohort Experience. J Parkinsons Dis 10, 665-675. 